\title{
BEITRÄGE DER TOPONOMASTIK ZUR LANDSCHAFTSGESCHICHTE (WALDENTWICKLUNG) UM LANGENTHAL
}

\author{
VALENTIN BINGGELI
}

\section{DIE MATERIALGRUNDLAGE}

Neben der Bedeutung, die Orts- und Flurnamen für Siedlungsgeographie und -geschichte haben, können sie interessante Hinweise geben auf weitere ehemalige Verhältnisse in der Landschaft, sowohl der Natur- wie der Kulturlandschaft. Namen haften oft erstaunlich fest an Ort und Scholle, überdauern nicht selten weit das Benannte. Sie vermögen sogar über den Bereich schriftlicher Quellen weiterzureichen und leisten damit wertvolle Dienste für urkundenarme Gegenden und Zeiten.

Gegenüber archäologischen Funden beispielsweise ist ein augenfälliger Vorzug, daß die Namen als Worte zu uns sprechen, allerdings mehr oder minder direkt (Sprachentwicklung, Umdeutungen, Erbstücke älterer Sprachen).

Eine Quelle der Ungenauigkeit liegt in ihrer Subjektivität: Die Namen sind im Blickwinkel ihrer Erzeuger, vor allem der bodentreuen Bauern, zu beurteilen. «Sie sind ein... lebendiger Spiegel, zwischen ihnen und den realen Gegebenheiten steht der Mensch, der sie geprägt hat» (Bandle, 1953. 146). Wir begegnen all den Schwierigkeiten nach Möglichkeit durch den fundierenden Beizug älterer Namenformen, einmal mundartlicher, vor allem aber urkundlicher Belege. "Die Erklärungen sollen grundsätzlich auf den älteren Belegen aufbauen, weil die heutigen Formen ja in der Regel das Ergebnis einer langen Entwicklung sind» (Schwarz, 1950. 14). Wir ziehen zudem soweit immer möglich die Angaben der Archäologie, der Pollenanalyse, der Forstwissenschaft usw. und solche alter Karten und Pläne heran. Gewisse Gefahren der Fehldeutung werden in der ortsnamengcographischen Forschungsrichtung dadurch vermindert, $\mathrm{da}$ sie nicht mehr «Raritätenkabinett» sein will, sondern «die Namen vor allem in ihrer Gesamtheit innerhalb eines bestimmten Gebietes betrachtet» (Bandle, 1953, 146).

Im folgenden sei als Beispiel das Zeugnis der Flurnamen für die Waldentwicklung in den bernischen Gemeinden Langenthal, Thunstetten und Kirchberg, vor allem der erstern, aufgezeigt.1 Einerseits bieten sich im kleinen Untersuchungsraum bedeutsame Vorteile der Vertiefung, der Detailkenntnis der landschaftlichen und lokalhistorischen Verhältnisse, der Ortsmundart und der Möglichkeit konsequenter Verfolgung urkundlicher Formen. Die Gefahrenquellen des engen Gesichtskreises anderseits gilt es zu überwinden durch Vergleiche mit andern Gebieten (Emmental, Thurgau, Voralpen), aus denen Bearbeitungen des Flurnamenguts vorliegen.

Mit Ausnahme von Kirchberg und Lützelflüh konnte angenähert der Gesamtnamenbestand, der auf Grund der Quellen möglich ist, (vgl. Anmerkung am Schluß) erhoben werden. Im Falle Langenthals sind es an die 600 Flurnamen, mit 27o urkundlichen Belegen, bei Thunstetten 44o Flurnamen mit ebenfalls 27o Urkundenformen. Zusammen mit den 430 Flurnamen der Kirchgemeinde Kirchberg BE standen demnach rund 1500 Namen zur Verfügung. Darunter befinden sich 300 Namen der Bewachsung (nicht eingerechnet die eigentlichen «landwirtschaftlichen» Namen), inkl. 8o Baumnamen. Bandle (1953) untersuchte im Thurgau 246 Namen von Einzelbäumen.

\section{DIE ZUSAMMENSETZUNG DES WALDES}

Unter den namenbildenden Erscheinungen steht die Bewachsung in unserem Gebiet an oberster Stelle, aber auch in den verglichenen alpinen, emmentalischen und oberrheinischen an hervorragendem Platz. Das Bild der Landschaft ist in den bewohnten Regionen stark durch das Vegetationskleid bestimmt. Bewach:ungsnamen helfen vor allem, die Verbreitung einzelner Pflanzen und des Waldes in früherer Zeit festzustellen, wobei allerdings sehr zu beachten ist, daß mithin nicht weite Verbreitung sondern Sel-

1 Kapitel einer unveröffentlichten ortsnamengeographischen Arbeit, betitelt: «Siedlungs- und Geländenamen als Zeugen der Natur- und der Kulturlandschaft.»Dargestellt anhand des $\mathrm{Na-}$ menbestandes von Langenthal und einiger Vergleiche mit Thunstetten, Kirchberg BE (beide im tieferen Mittelland), Lützelflüh (höheres Mittelland), Leißigen, Därligen (Voralpen) und Diersheim (rechtsrheinische süddeutsche Gemeinde). 
tenheit eines Gewächses zur Namengebung angeregt hat. So fiel bei uns die Linde nicht durch Häufigkeit, sondern durch die Besonderheit als Gerichts- und Grenzbaum auf. ${ }^{2}$

Pollenanalytische Bodenprofile, als exakte Stützung der toponomastischen Ergebnisse, liegen vom Burgäschisee vor (Welten, 1947); ihnen entnommen sind die Grundlagen zu Fig. 5. Die Zahlen des Vorkommens von Bäumen in Flurnamen sind in den Fig. 3 und 4 verarbeitet. Als ungefähren mittleren Zeitpunkt, für den das Namengut Zeugnis ablegen dürfte, wurde 1400 eingesetzt, da das geschichtlich von Meyer (1943 u. a.) ausgewertete st. urbanische Urbarmaterial besonders von 1464, sodann von 1194, 1277, 1303, 1530 und 1562 stammt.

Erstaunlich ist die Übereinstimmung der Fig. 3 bis 5. Sie zeigen, vergleichen wir sie mit Fig. 1 und 2, wie stark sich auch bei uns die pflanzlichen Verbreitungsverhältnisse seit dem Mittelalter, d. h. seit der Namenentstehung, verändert haben, was für Mitteleuropa allgemein bekannt ist. Es zeigt sich als erstes ein starkes früheres Überwiegen der Laubhölzer über die Nadelhölzer. Der natürlichen Vegetationsregion nach gehört in der Tat das tiefere Mittelland in eine vorwiegend mit Laubholz bewachsene Zone, der Oberaargau in jene der Buche und Eiche mit hügelwärts zunehmender Weißtanne. Auf Grund der Flurnamen kann gesagt werden, daß sich das Verhältnis Laubwald : Nadelwald seit dem Mittelalter fast in den reziproken Wert verändert hat.

\begin{tabular}{llrlllll} 
& & Buche und Eiche & $:$ & Fichte und Tanne \\
\hline Heute: & Oberaargau & 5 & und & 3 & $:$ & 49 & und 28 \\
(Holzarten in Prozent) & Thurgau & 10 & und & 1 & $:$ & & $(80)$ inkl. Föhren \\
Früher: & Oberaargau & 14 & und & 16 & $:$ & 10 \\
(Flurnamen-Anzahlen) & Thurgau & 20 & und & 20 & $:$ & 10
\end{tabular}

Laut Statistik der Forstverwaltungen ${ }^{3}$ stehen heute im Oberaargau und Thurgau ziemlich genau gleicherweise den rund $80 \%$ Nadelhölzern ${ }^{4}$ rund $10 \%$ an Buche und Eiche in der Holzartenzusammensetzung des Waldes gegenüber. Auf Grund der Flurnamenhäufigkeit ergeben sich für das Mittelalter (um 1400) in den genannten drei oberaargauischen Gemeinden 13\% Nadelholz neben 30\% Buche und Eiche. Weiter sind namenbildend vertreten: Birke 14\% ( Die lokal zahlreichen flachen Sumpfböden trugen durch die ganze Zeit 7 bis 20 bis 40\% Birke»: Welten 1947, 109; anhand Pollendiagramm Burgäschi), Erle 10\%, Weide 9\%, Esche/Hasel/Linde je 5\% und Föhre 4\%. Die Zusammensetzung wird, vor allem was die Nadelhölzer betrifft, durch die Pollenanalyse bestätigt (Welten, 1947, vgl. Fig. 5), ebenso durch die Thurgauer Zahlen Bandles (1953), denen ein zahlreicher Namenbestand zugrunde liegt.

$\mathrm{Zu}$ betonen ist, daß auch die Hard-Namen auf Eichen hindeuten. Nach K. A. Meyer (1931) bedeutete Hard früher fast ausschließlich Laubwaldung und zwar besonders Eichen-Weide-Mischwald. Von den Eichennamen kommt nur einer ins Gebiet des großen Hardwaldes (Langenthal) zu liegen. So darf in der Tat angenommen werden, die Eiche hätte in unserer Gegend früher eine dominierende Rolle gespielt, zusammen mit der Buche, die der Pollenanalyse nach im Mittelalter jahrhundertelang die Eiche übertraf, im Landschaftsbild aber minder auffiel und so weniger Namen anregte.

2 Sichere Angaben bedeuten Namen von Pflanzen, die heute in dem Gebiet äußerst selten oder nicht mehr vorkommen. Für Skandinavien haben wir das Beispiel von Boket, Böket, bok = Buche, das zusammen mit pollenanalytischen Befunden zeigt, daß die Buche dort einst bis $300 \mathrm{~km}$ nördlichere Verbreitung aufwies (berechnet aus Karte in Bandle 1953, 158; Quelle: Bertil Lindquist, Den skandinaviska bokskogens biologi. Svenska Skogsvardsföreningens Tidskrift, $3,1931)$.

3 Wir verdanken die Angaben dem Kreisforstamt X in Langenthal (Oberförster M. Ammon) und der Forstverwaltung Langenthal (Oberförster P. Meyer).

4 Fichte (Rottanne, Picea exelsa), Tanne (Weißtanne, Abies alba), Föhre (Kiefer, Dähle, Pinus silvestris). 
Heutige Holzartenzusammensetzung des Waldes

Figur 1: Oberaargau

Figur 2: Thurgau

(nach den Forstverwaltungen)
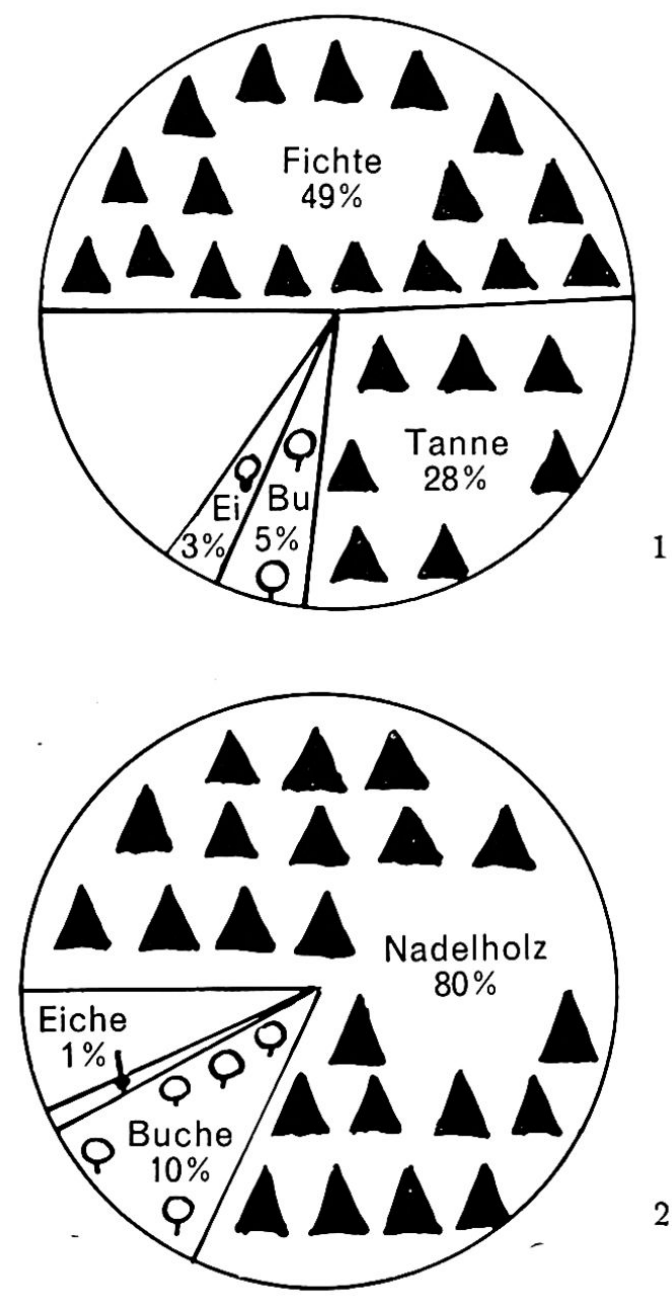

2
Holzartenzusammensetzung des Waldes utm etwa 1400

Figur 3: Oberaargau (nach Flurnamenanzahl) Figur 4: Thurgau (nach Flurnamenanzahl) Figur 5: Pollenanalyse Burgäschisee

3
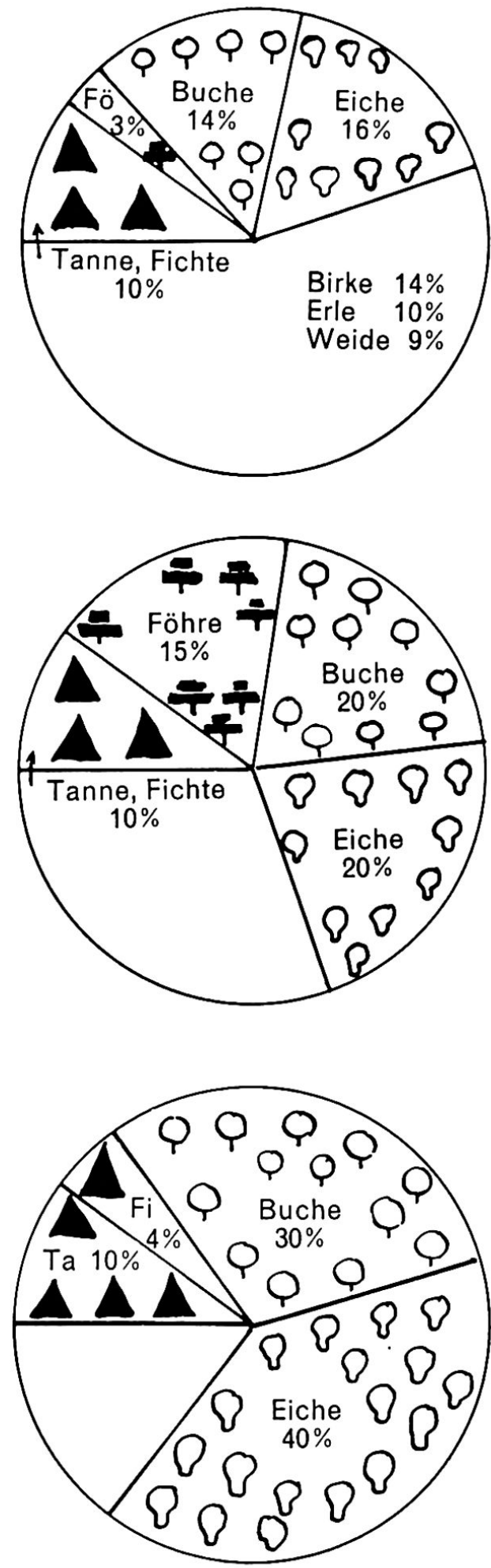

5

Figur 1: Oberaargau; d. h. die oberaargauischen Gemeinden Langenthal, Aarwangen, Lotzwil, Melchnau, Roggwyl, Wynau, Herzogenbuchsee, Thunstetten, Bußwil, Obersteckholz, Bleienbach, Madiswil, Rütschelen und Schwarzhäusern

Figur 2 und 4: Zahlen approximativ, berechnet nach Bandle (1953)

Figur 3: Gemeinden Langenthal, Thunstetten und Kirchberg $\mathrm{BE}$

Figur 5: Approximative Werte aus Diagramm Welten (1947) 
Diese beiden Baumarten sollen schon zur Römerzeit häufig vertreten gewesen sein. «Die Römer berichten hauptsächlich von Eichen- und Buchenwäldern» (Guyan, 1954. 221). Im Mittelalter wurden sie der Waldweide wegen vorgezogen. «Ums Jahr 500 hieß das Gesetzbuch der Burgunder nur Eichen und Buchen als die acherumliefernden Bäume, «arbores fructiferae» (Acherum, got. Akran - Frucht: Eicheln und Buchekker), während Föhren und Tannen als weniger geschätzte Waldbäume galten» (Guyan 1954, 221).

Daß die frühere Zusammensetzung der Wälder stark auf die Laubholzseite neigte, kann außer mit Hard noch mit weitern Namen bestätigt werden. Hürschi deutet auf Gebüschwald hin (siehe Kap. 3). Löli, ev. auch Loch, von ahd. loh, mhd. loch, lat. lucus = Hain, Wald. Nach Schwarz (1950, II, $177 \mathrm{ff}$ ) ist für Deutschland die Bedeutung «lichter mit Eichen und Buchen bestandener Wald» gesichert.

Was Nadelholz-Namen betrifft, haben wir uns näher zu betrachten: 1269 toub welden. In einer Urkunde von 1269 betreffend das Kirchspiel Thunstetten ist von «vranwelden vel toubwelden» die Rede. Nach Hubschmied (1938) sind «Tob-, Topp-, Doppel-, Dau-, Taub-, Taugwald Waldnamen in den Kantonen Bern und Wallis; in der ältern Sprache lebte tobwald noch als Appelativ für die großen dunklen Tannenwaldungen (silvas nigras que theotonice wulgo tob welde appellantur, 1299)», dunkle Wälder, die in der deutschen Volkssprache tobwelde heißen.

Hubschmied erklärt die Form vom erschlossenen gall. dubo - schwarz her (dubo juris oder juris duba - Schwarzwald). Das «Quellenwerk zur Entstehung der Eidgenossenschaft» (I, 462, zit. Meyer o. J.; b) indessen ist der Meinung, es handle sich um Fronwälder und dürre abgestorbene Wälder; von mhd. toub - abgestorben, trocken, dürr.

Das sagenhafte Adelmännli in der noch heute mächtigen Waldung gegen St. Urban zu läßt etwas ahnen von unheimlich dunklen Forsten von vorzüglich Nadelhölzern. Visà-vis des Adelmännli übers Rothtal heißt es: Sagenchöpfli. Es ist die bei Gotthelf berühmt-berüchtigte Gegend dichter, schauerlicher Wälder, die bereits für das 15./16. Jahrhundert in der Grenzgegend zwischen Ober- und Unteraargau Erwähnung finden (Meyer 1943). Dichter Hochwald mit Nadelhölzern dürfte in unserer Gegend also bereits damals bestanden haben neben ausgedehntem, lichtem Laubwald, der Waldweide (1826 in Langenthal abgeschafft). Der Weidgang aber gerade war es, der den laubbeholzten Allmendwäldern argen Schaden zufügte. Wohl wurden Eichen und Buchen des Acherums wegen gehütet und den Tannen vorgezogen. Das anspruchslosere, rascher wachsende Nadelholz hatte dagegen günstigere Voraussetzungen, wurde zudem in der Neuzeit durch planmäßige Forstwirtschaft gefördert. Sein Vordringen mag auch durch die Klimadepression (Gletscherhochstände) um 1600 beeinflußt worden sein.

Stark zum Laubholzrückgang beigetragen hat das mittelalterliche «Zäunen» und «Hagen». Jedes Haus war palisadenartig eingefriedet, um das Dorf zog sich der Etter, die Felder mußten nach Gemeindesatzung eingezäunt werden. Im Langetental erforderten die Wässermatten «Wuhrhölzer», «Brütschestüd» usw. «früher immer eichigi» (Friedli 1925, 84). Von der Mitte des 19. Jahrhunderts an benötigten Käsereien und Bahnbau (Eichenschwellen) Unmengen an Starkhölzern, abgesehen vom Bedarf der wachsenden Bautätigkeit allgemein.

Es erhellt deutlich, daß der Mensch neben Rodung und Melioration auch die bedeutende landschaftsgestaltende Veränderung der Zusammensetzung des Pflanzenkleides zum großen Teil verursachte.

\section{DIE EHEMALIGE AUSDEHNUNG DES WALDES}

Art und Ausdehnung des Waldes prägen einen der bedeutendsten Charakterzüge im Antlitz der Landschaft, früher ungleich mehr noch denn heute. Unser Land im Flugbild ist einem Tuche zu vergleichen, der helle Grund des Wies- und Ackerlandes im Laufe der Jahrhunderte mit Bauernfleiß gewoben, verteilt darin als mannigfaches 


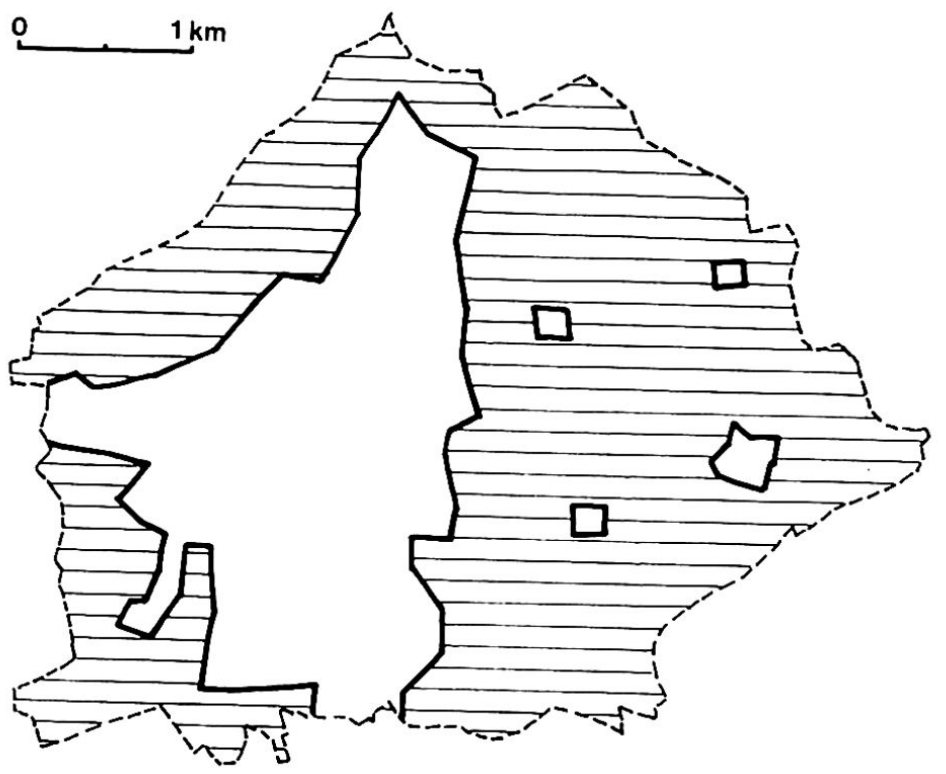

Figur 6: Gemeinde Langenthal. Ausdehnung des Waldes um ca. 1400 (rund 850 ha), rekonstruiert auf Grund von Flurnamen und urkundlichen Angaben

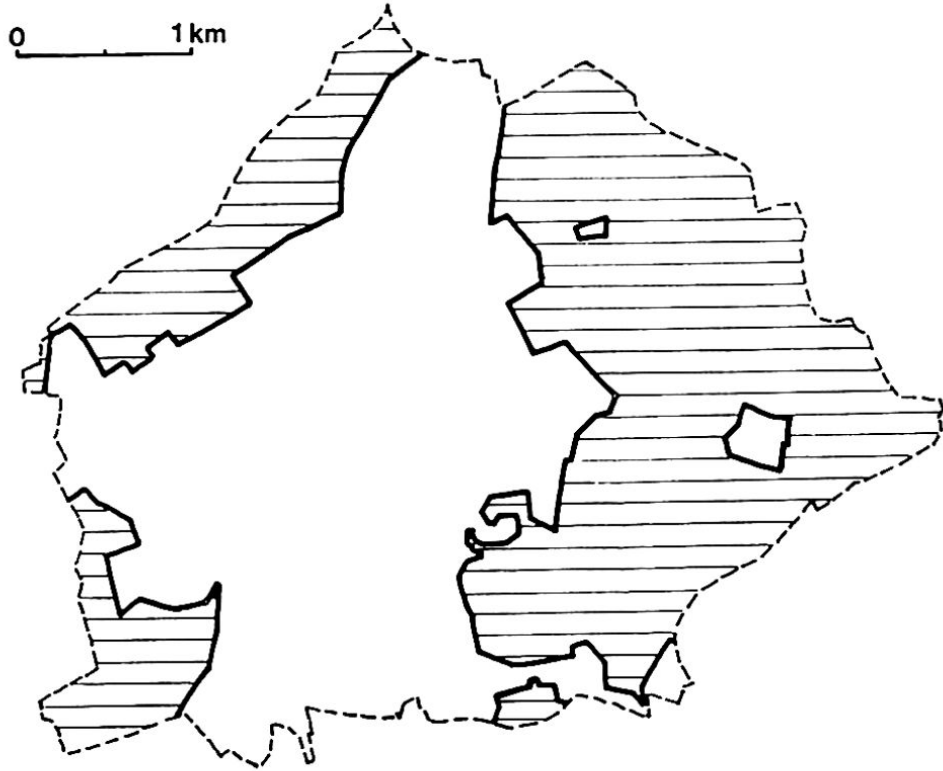

Figur 7: Gemeinde Langenthal. Waldausdehnung nach dem "Plan von Langenthal» von 1810 (rund $730 \mathrm{ha}$ )

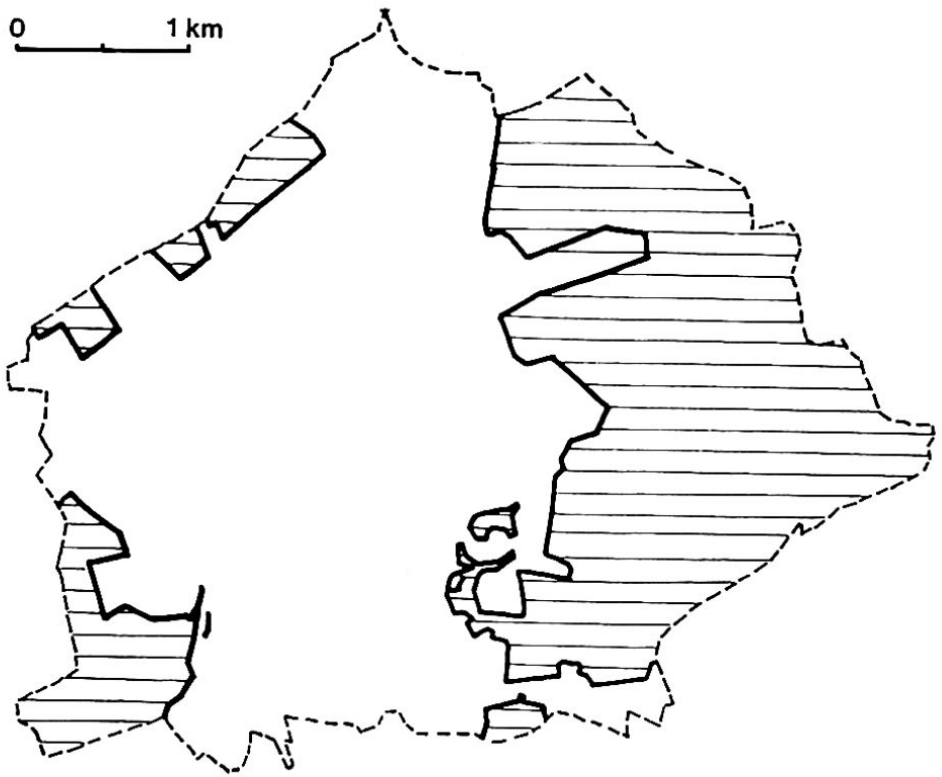

Figur 8: Gemeinde Langenthal. Die heutige Waldfläche nach dem Grundbuch-Übersichtsplan 1949 (664 ha um 1942; Gesamtbodenfläche der Gemeinde: $1143 \mathrm{ha}$ ) 
Ornament der Wald. Die Umwertung vom frühern Waldreichtum zum heutigen gehegten und gepflegten und forstgesetzlich vor Verminderung geschützten Wald- und Holzbestand erhellt trefflich eine Stelle aus den Akten des großen Zehntprozesses von 1809-12 zwischen Langenthal und dem Kloster St. Urban, wo Fürsprech Me:smer schreibt: «Das Holz hatte an sich wegen seiner Menge einen geringen oder beinahe keinen Wert» (Meyer 1943).

Die Rekonstruktion der alten Naturlandschaft können nur systematische pollenanalytische Bodenuntersuchungen ermöglichen. Daß aber das Landschafts- und Waldbild noch im Mittelalter ein weit vom heutigen abweichendes war, das beweisen auch die Flurnamen. So liegen von denjenigen, die mit dem Wald enge Beziehungen haben (eigentliche Waldnamen, Wald- und Allmendwirtschaftsbegriffe, Rodungsnamen) bloß 30 innerhalb der heutigen Waldungen, ganze 62 aber außerhalb! (Fig. 9).

Nicht alle Namen haben dieselbe Aussagekraft. Brauchbar sind vor allem Beständenamen wie Buechrain, Erlihalde, alle -holz. Aber auch mit i endende Namen von Einzelpflanzen, Hasli, Aspi, Erli, Dennli, können ähnliche Bedeutung haben, wenn sie zurückgehen auf das ahd. Kollektivsuffix -ahi (z. B. Buechahi - Buechi - Buchengehölz) (Schwarz 1950, II, 80; Hubschmied 1938, 731). Auf Bestände hin weisen auch: Gurten, Hard, Löli usw. (Erklärung des letztern siehe vorn). Der oder das Hard ist die ehemals ungleich größere Waldung im Nordosten Langenthals, auf die auch «Gurtenen» hinweisen mag; von gall. juretton - Wald (zu franz. Jorat) ; juris duba - Schwarzwald (Hubschmied, 1938). Hard ist in Deutschland ein eigentlicher Waldname (Schwarz, 1950, 178), die berühmtesten derartigen Namen sind Harz und Hardt. Ähnlich dürften unsere Flurnamen Schorrerhard, 1562 und Thunstetterhard, $1530 \mathrm{zu}$ deuten sein. Das Hard ist der urkundlich älteste Wald Langenthals: 1194 Hard, 1277 Harde, 1303 Hart, 1464 Hard, 1562 Hartt.

Auch das Idiotikon (IV, 1595) erklärt den Namen als eng mit dem Wald verbunden: a) mhd. hart - fester Sandboden, Weidetrift, Wald. Gemeintrift, Almänd, g'mein Gut, compascuum. Wort meist von Waldungen gebraucht oder von früher bewaldet gewesenen zum Teil Feld gewordenen Gegenden. b) Bezeichnet immer einen größeren, der Gemeinde gehörenden Wald; ev. ursprünglich das als Gemeindeweide dienende Brachfeld, das man zeitweise oder später dauernd zu Wald werden ließ, wenn der Boden wirklich zu «hart» zum Ackerbau war und vielleicht von dieser Eigenschaft den Namen bekommen hatte.

Für das 14./15. Jahrhundert sind uns die Langenthaler Wälder ziemlich vollständig bekannt, allerdings zumeist bloß in ihren Namen und weniger in den genauen Ausdehnungen. Einige Unterstützung bietet die Schœpf-Karte von 1572, die nachgewiesenermaßen in unserem Gebiet auf gut 2 Jahrhunderte frühere Verhältnisse zurückreicht (Verfasser 1957). Schoepfs Kenntnis unseres Gebietes beruht demnach wohl auf älteren Quellen, die Karte scheint allgemein in diesem Sektor wenig präzis und verläßlich. Die weite Waldung östlich Langenthals ist bei ihm ein rudimentäres Wäldchen. Was eher zuträfe bei Schœpf, ist der langgezogene Waldgürtel im Westen, ein an die 10 Kilometer langer «Hardwald»zwischen Schorenterrasse und Murgenthal. Eine solche Waldung wird in der Tat auch durch vereinzelte Flurnamen angedeutet.

Von den 16 Wäldern des Urbars von 1464 (St. Urban) konnten Ratissegg und Georgenholtz nicht lokalisiert werden. Es sind : (ein Wald heißt) der Greppen, an der Erlihalden, das Aspe, die Schwende, der Hinterberg, das Eichholtz, in Betten, in Lüttisswinckel, Ratissegg (oder Retisegg), Hochhärdli, Niderhard, Oberhard, Georgenholtz (oder Jörgenholtz), Wischberg (oder Wisberg), Adelmännly; ein Wäldlein heißt die Bachspreitte (oder Bachspreitti). Dazu kommen weitere wäld, holtz und holtzli, bezeugt für 1530 und 1562 .

Hürschi (1530 Hürschacher) möchten wir von Hurst, Hurscht ableiten, was «in älterer Sprache Gebüsch, Gesträuch» bedeutet (Hubschmied 1938, 736). Hurst heißt z. B. ein Wald bei Hindelbank, Hursch ein Wäldchen bei Jegenstorf. 
Auch schwartz egerten 1464 (Schwarz Egerten 1530) darf nach der Deutung des Idiotikons bedingt als Bezeichnung einer einmal bewaldet gewesenen Stelle betrachtet werden. Ähnlich verhält es sich mit den Allmenden (Waldweide), die oben besprochen wurden.

Von 1810 stammt das erste kartographische Verzeichnis unserer Wälder, im «Plan von Langenthal von 1810» des Heimatmuseums Langenthal. Auch in den zwischen dem 15.und 19. liegenden Jahrhunderten sind, wie daraus zu ersehen ist (vgl. Fig. 6 und 7), «große Stuke des Holzes entblöst worden». Sogar nach 1800 wurde hier noch recht kräftig gerodet, wenn wir die Waldarealzahlen vergleichen: 1810 rund 730 ha, 1942 noch 664 ha. $\mathrm{Ob}$ unser Gebiet gegenüber der allgemeinen Ansicht, nach dem Mittelalter sei die Rodungstätigkeit weitgehend abgeschlossen gewesen, eine vereinzelte Ausnahme macht? Jedenfalls ist auch die Hauptrodungszeit ( vgl. nächstes Kapitel) für den Oberaargau um etwa 2 Jahrhunderte später als allgemein üblich anzusetzen, auf die Zeit von 1200 bis 1400 .

\section{DIE WALDRODUNG}

Wie Art und Ausdehnung des Waldes einen der bedeutendsten Charakterzüge des Landschaftsgesichtes darstellen, so ist die Rodungstätigkeit der stärkste landschaftsgestaltende Eingriff des Menschen. Zu verschiedenen Zeiten kam es auch zu eigentlichen Raubrodungen, zur Dezimierung von Waldbeständen, die aus bekannten lokalklimatischen Gründen und vor allem denen der Bodenerosion katastrophale Folgen zeitigten. Wie diese weltweiten, jahrtausendjährigen Probleme im großen, so zeigen auf kleinem Raum nicht minder deutlich die Flurnamen die hervorragende Stellung des Waldes und der Waldrodung im Leben der Landschaft und der Menschen.

Das vergangene Jahrtausend ist die eigentliche Rodungszeit der Menschheitsgeschichte. Für unser Gebiet zeigt sich, daß nach der allgemeinen Hauptrodungszeit um 1000 auch im spätern Mittelalter, ja in den letzten anderthalb Jahrhunderten stark gerodet wurde. Rodmann ist ein Langenthaler Geschlecht, dessen Name für das 16. Jahrhundert bezeugt ist! Heute ist diese Rodezeit Vergangenheit. Alle Rodungsnamen haben ahd. Ursprung. Was vor der Landnahme der Schweiz durch die Alamannen in der Schweiz (5. Jahrhundert) gerodet wurde, entgeht weitgehend unserer Kenntnis. $\mathrm{Da} \beta$ schon die Römer, vorher Latène- und Hallstattleute Ackerland dem Walde abgerungen haben, ist sicher. Für diese frühesten Rodungen können uns archäologische Fundstellen gewisse Auskunft geben. Wir wissen, daß schon in keltischer Zeit das schweizerische Mittelland relativ dicht besiedelt war (aus Cäsar bekannt die 12 Städte und 400 Dörfer der Helvetier).

Nach Wander-, Landnahme- und Zeit der frühen alamannischen Seßhaftigkeit folgt ungefähr mit 700 der eigentliche Landesausbau und damit die Rodungstätigkeit. Denn mit der Bevölkerungsvermehrung wuchs parallel der Bedarf an Ackerfläche. Ausgehend von der Eigenart der germanischen Stämme, in Einzelhöfen, nicht in Dörfern zu siedeln, nimmt man an, es seien inselartig im immer noch weit das Landschaftsbild beherrschenden Walde die Stellen der verstreuten Höfe gereutet worden.

Rodungsnamen treten urkundlich erst mit dem Kloster St. Urban, gegründet 1194, auf. Fehlen zu den im folgenden aufgeführten Flurnamen die urkundlichen Belege, so besteht natürlicherweise die Möglichkeit, daß sie ein höheres Alter haben, eher jedoch, daß sie bedeutend jünger sind. Die ahd. Form kann sehr lange lebendig geblieben sein. Klarer wird die Aussage, wenn zeitlich festgelegt werden kann, wann der Begriff nicht mehr verstanden wurde.

Schwändi (und dazu gehörend Schwändibächli, Schwändihölzli) (1336, 1464 Schwende; 1530 Schwendi, Schwendematten). Schwenten (ahd. swant) - schwinden machen, den Wald zum Schwinden bringen (Id. IX, 1928-49; Hubschmied 1938, 740). 
Brodmatt könnte rod-matt geheißen haben und bedeuten, trotz Friedli (1925, 249), der Brotmatt schreibt. Für unsere Deutung spricht folgendes: die Brodmatt liegt 1. in einem ausgesprochenen Waldeinschnitt und zweitens in unmittelbarer Nachbarschaft der Schwändi. Roden wie reuten bedeuten das Schlagen von Waldstücken zum Ziele der Gewinnung von zusätzlichem Ackerland. ahd. riuti und rod stehen im Ablauts-verhältnis zueinander, ähnlich wie nhd. sieden und gesotten (Hubschmied 1938, 749).

Sängeli (Thunstetten), Sängi (Obersteckholz) (1530 Sänge, 1562 Sengiholtz, 1578 in der Sengen [Schoepfkarte]), ev. Sandbächli, -brünneli (Nähe Riedhof) von Sand, Sang, sengen (ahd. sangjan, mhd. asangen) von brennen; Wald durch Feuer zum Schwinden bringen. (Dazu: Brandholz [Bleienbach] 1530 Brandtholz.)

Riedhof: 1260 viculus Riede; Kuno von Ried, erwähnt 1224 (Meyer). Nach Schwarz (1950, II, 180 f.) ist die Scheidung von ahd. riot (riuti) = Rüti und ahd. hriod = Ried, mit Sumpfgras bewachsener Boden, schwierig. Unser Ried (hof) allerdings geht eindeutig auf das erste zurück: ahd. riodan (Verb), riot, riod $=$ Rodung. (Id. IV, 1730; VI $1731 \mathrm{f}$.; Schwarz 1950, II, 268). Riedhof ist heute der Name für ein Waldstück auf Anhöhe Pt. 529 des Grundbuchübersichtsplanes 1949. Noch auf dem «Plan von Langenthal» von 1810 aber ist hier eine an die 20 Jucharten haltende Lichtung verzeichnet (Karte Fig. 6, 7).

Stockmatten in den nidren Matten 1562. Nach Schwarz (1950) handelt es sich bei Stock- ebenfalls um einen alten Rodungsnamen.

Mit der Gründung des Klosters St. Urban 1194 setzen wir den Beginn der Hauptrodungszeit an, die vor allem etwa die nächsten beiden Jahrhunderte umfaßte. Den Zisterziensermönchen, deren Kloster bald die mächtigste Grundherrin der Gegend war, war von der Ordensregel aus die Urbarisierung des Bodens geboten. In Deutschland waren sie in der Rodungstätigkeit führend (Schwarz 1950. II, 182). Bei uns sind sie in hervorragender Erinnerung durch die Kanalisierung der Langeten in Richtung Roggwil, die damit verbundene Entsumpfung des Geländes und die Anlage der Wässermatten. Daß sie aber ebenfalls verantwortlich sind für eine ausgedehnte Reut-, Brand-, Sang-, Stock- und Schwentarbeit, belegen Flurnamen und Urkunden. Eine große Anzahl Namen der Urkunden von 1464, 1530, 1562 und weitere, die der Volksmund überliefert hat, haben ihren Ursprung in der Dreifelderwirtschaft des Mittelalters. (Da für die Zeit vor den erwähnten Jahren keine derartigen Namen bezeugt sind, nehmen wir sie bei uns alle als (höchstens) im hohen und späten Mittelalter entstanden an. Von diesen Namen berichten einige ebenfalls vom Schlagen des Holzes. Die Allgemeinweide, die Allmend, war nämlich bewaldet, d.h., besser, umgekehrt: die Wälder überhaupt machten, wohl mit wenigen Ausnahmen, die Allmend aus. Einzelnen wurde darin Stücke zu roden bewilligt, wovon die Ischläg, Bifang, Bünten berichten.

Über Alter und Ausmaß der Rodungen haben wir durch Namen und Urkunden einiges erfahren, es bleiben nachzutragen Angaben der Namen über die Rodungsart. Die einfachste aber seltenste ist das Brand- oder Sang-Vorgehen (Brandholz, Sängeli, Sängi). Das Sengen, Brennen (ahd. sangjan, mhd. asangen) stellt einen groben, unrationellen Eingriff dar, einzig der Vorteil eines aschengedüngten Bodens lag vor. Diese Art soll vorwiegend in Buschwäldern zur Anwendung gelangt sein.

Verschiedenartige Vorgehen bezeichnen weiter Schwändi einerseits und Rüti/Ried/ Rod andererseits. Alle scheinen in frühen Rodungszeiten schon angewandt worden zu sein, haben doch sämtliche Namen ihre ahd. Ursprungsform. Beim Schwand, ahd. swant, schwenten - schwinden machen, den Wald zum Schwinden bringen durch Entrinden der Stämme (Id. IX 1928; 49. Hubschmied 1938. 740), indessen beließ man einfachheitshalber die Baumstrünke im Boden, ließ sie also derart direkt wieder ihrem neuaufbauenden $Z$ wecke zukommen. Beim Reuten (ahd. riodan, riot, riod) (Schwarz 1950. 268) dagegen wurden die Strünke und Wurzeln «ausgemacht» 
$\circ$

$\Delta$

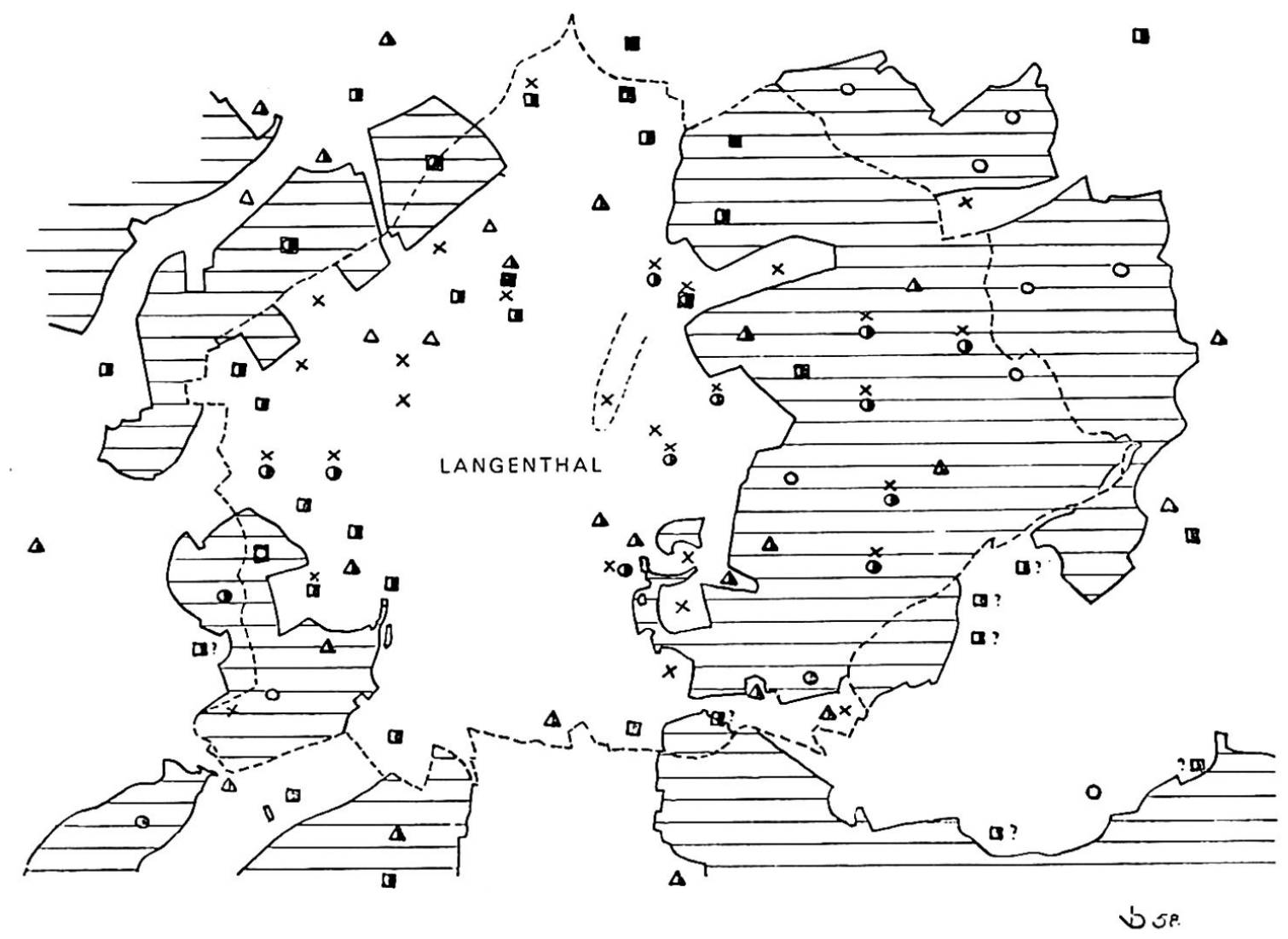

Figur 9: Die Wälder um Langenthal (schraffiert) und die Streuung der mit Bewaldung zusammenhängenden Flurnamen. Die Namen außerhalb des heutigen Waldareals, zumal diejenigen entlang den augenfällig künstlich ausgezackten Waldsäumen, spiegeln in ihrer Geschlossenheit zum Teil geradezu eine ehemalige Ausdehnung des Waldes

Signaturen :

Kreis: Namen heutiger und ehemaliger Wälder. Rechteck: Flurnamen, die auf ehemalige Bewaldung hindeuten (z. B. Hard, Hurst, Loh). Dreieck: Rodungs-Flurnamen (z. B. Schwändi, Sängi, Rüti). Die einzelnen Formen siehe Text. Kreuz: Urkundlich bezeugt bewaldete Stelle. Signatur ausgefüllt: Namenursprung vordeutsch. Signatur halb ausgefüllt: Namenursprung mittelalterlich oder Name urkundlich bezeugt. Signatur offen: Anscheinend jüngerer Flurname

(Id. IV, 1730; VI, 1371 f; Hubschmied 1938. 739). Es dürfte auch Stock(matte) hiezu gehören.

Leistung und Arbeit der Rodenden werden heute leicht zu gering eingeschätzt. In Wirklichkeit war der Reuterschweiß geradezu der erste Regen, den das neuerschlossene Ackererdreich empfing. Während des letzten Krieges konnte man noch als Augenzeuge des ganz ungewöhnlichen Eindrucks eines größern Reutwerkes teilhaftig werden: ein zähes Riesenringen, ein urweltliches Wurzelgewirr, ein gewaltiges, gewaltsames Auferstehen erdarmiger Kobolde der Tiefe. Die mittelalterlichen Herrscher bezahlten oft die schwere Arbeit mit dem gesengten, geschwenteten, geschlagenen, gerütteten Landstück (Neubruchs-, Kolonistenrecht) oder mit der Lösung der Leibeigenschaft. J. R. Meyer vermutet, die Herren von Ried, deren viculus im großen Forst östlich Langenthals bereits im 13. Jahrhundert erwähnt wird, seien solche Rodungssiedler, Rodungsfreie gewesen. 


\section{ZUSAMMENFASSUNG}

Anhand von Flurnamen einiger bernischer Gemeinden, vor allem Langenthals, wird die ehemalige Zusammensetzung und Ausdehnung des Waldes landschaftsgeschichtlich untersucht. Unerläßlich ist bei solchen orts- und flurnamengeographischen Studien der Beizug urkundlicher Belege (alte Namenformen), zudem die Fundierung durch naturwissenschaftliche, lokalhistorische und altkartographische Befunde.

Für das Gebiet der untern Langete ist die Hauptrodungszeit (allgemein 1o.-12. Jahrhundert) auf 13./14. Jahrhundert anzusetzen (Kloster St. Urban). Nach den vorgelegten Karten, basierend auf Flurnamen- und Urbarangaben, wurden aber auch nach dem 14. Jahrhundert erheblich große Waldflächen gerodet: Nachdem der Wald um 1400 eine Fläche von rund 850 ha gehalten hatte, waren es 1810 noch 730 ha, 1942 noch 664 ha.

Die mittelalterliche Zusammensetzung der oberaargauischen Wälder in bezug auf Baumarten bezeugen die Flurnamen in guter Übereinstimmung mit der Pollenanalyse. Die Bearbeitung des zahlenmäßig reichen Thurgauer Materials ergab ebenfalls unterstützende, sehr ähnliche Resultate. Die Wälder der studierten oberaaragauischen Gemeinden setzen sich heute aus rund $80 \% \mathrm{Nadel}$ holz und 10\% an Buche und Eiche zusammen. Die Häufigkeit der Flurnamen von Einzelbäumen läßt für die Zeit von 1400 auf bloß wenig mehr denn 10\% Nadelholz gegenüber 30 bis $40 \%$ Buche und Eiche schließen, was durch die Pollenanalyse weitgehend bestätigt wird (14\% Nadelholz, 70\% Buche und Eiche). Auch Birke, Erle und Weide sind in Flurnamen dieser Zeit annähernd so häufig vertreten wie Eiche und Buche.

\section{LITERATUR}

1. E. Schwarz: Deutsche Namenforschung. Band I Personennamen, Band II Orts- und Flurnamen, Göttingen 1950.- 2. A. Bach: Deutsche Namenkunde II, 2 Bände, Heidelberg 1953/54. 3. A. Helbok: Die Ortsnamen im Deutschen, Berlin 1939. - 4.M.R. Buck: Oberdeutsches Flurnamenbuch, Bayreuth 1931. - 5. W. Bruckner: Schweizerische Ortsnamenkunde, Basel 1945. 6. P. Zinsli: Grund und Grat. Die Bergwelt im Spiegel der Schweizerdeutschen Alpenmundarten, Bern 1946. - 7. P. Ocrtli: Deutschschweizerische Ortsnamen, Erlenbach-ZH, o.J. - 8. Schwcizerisches Idiotikon. Wörterbuch der Schweizerdeutschen Sprache. Frauenfeld 1881 (zit. Id). 9. J.U.Hubschmied: Sprachliche Zeugen für das späte Aussterben des Gallischen. Vox Romanica 3, 1938 ; 1o. derselbe: Über Ortsnamen des Amtes Burgdorf... Heimatbuch Burgdorf Bd. II, Burgdorf 1938. - 11. O. Bandle: Die Naturlandschaft im Lichte der Flur- und Ortsnamen. Mitt. Thurg. Natf. Ges., Heft XXXVII, 1953. - 12. E. Fricdli: Aarwangen. Bärndütsch als Spiegel bernischen Volkstums, Bern 1925. - 13. R. Schedler: Wanderbuch für Oberaargau und Unteremmenthal, Bern 1925. - 14. P. Kasser: Geschichte des Amtes und des Schlosses Aarwangen. AHV, XIX. Bd., Bern 1908. - 15. F. Ramseyer: Wanderbuch Oberaargau, Bern 1956. - 16. J. R. Meyer: Über Geschichte und Namen der Langenthaler Wälder (versch. Artikel), Langenthaler Tagblatt vom 28. August $1943 ; 17$. derselbe: Im Allmen und auf Schoren. Beilage zum Langenthaler Tagblatt Nr. 22/23, Mai/Juni 1957; 18. derselbe: Über Herkunft und Bedeutung des Namens Langenthal. Mss. Gemeindearchiv Langenthal (o. J.; a) ; 19. derselbe: Flurnamen Langenthals. Unvollendeter Vortrag, Mss. in oben (o.J.; b) ; 2o. derselbe: Vom Entenmoosweiher, von der Gutenburg, von den Herren v. Ried und der Adelheid von Hurun. Mss. in oben. - 21. V. Binggeli: Über Qualität, Entstehung und Autorschaft der Anonymen Bernerkarte von 1749, Geographica Helvetica 3, 1957; 22. derselbe: Die Landschaft im Lichte der Flurnamen, Langenthaler Heimatblätter 1961; 23. derselbe: Das Dorf und seine Landschaft als heimatkundlicher Wohnraum. Führer durch Jubiläumsausstellung Langenthal $1961 ; 24$. derselbe : Über Begriff und Begrenzung der Landschaft Oberaargau. Jahrbuch des Ob. 1962. 25. H. Wahlen: Die Flurnamen der Gemeinde Lützelflüh, Bern 1943. - 26. E. Kohler: Alt Langenthal, Langenthal 1932. — 27. A.Kümmerly und O. Breiter: Heimatbuch von Thunstetten, Bd. II; L'thal 1957. - 28. K. Zollinger: Das Wasserrecht der Langeten. Diss. Bern 1906. - 29. W. Bicri: Die Wässermatten von Langenthal. Mitt. Natf. Ges. Bern, NF. 6. Bd.; Bern 1949. - 3o. M. Welten: Pollendiagramm Burgäschi. Ber. Geobot. Inst. Rübel in Zürich f. d. J. 1946; Zürich 1947. - 31. K. A. Meycr: Geschichtliches von den Eichen in der Schweiz. Mitt. Schweiz. Zentr. anst. f. d. forstl. Vers.wesen, XVI. Bd. 2, Zürich 1931. - 32. J. J.Frïh: Geographie der Schweiz, St. Gallen 193o-38. - 33. W. U. Guyan: Mensch und Urlandschaft der Schweiz. Zürich 1954. - 34. A. Heim: Geologie der Schweiz, Leipzig 1919 -22. - 35. Geologischer Führer der Schweiz, Einführungen, Basel 1934. - 36. Geologische Generalkarte der Schweiz Bl. II, 1:200 000. - 37. J. Kopp: Zur Geologie von Langenthal. Langenthaler Heimatblätter 1935.

\section{QUELLEN}

Nomenklaturkommission des Kantonalen Vermessungsamtes Bern. - Grundbuchübersichtsplan der Gemeinde Langenthal (1949). - Eigene Aufnahmen an Ort und Stelle. - Burgerarchiv Langenthal (Arbeiten J. R. MEYERs) : Urbar-Material. - Nomenklaturpläne und Namen-Kartothek der Ortsnamensammlung des Kantons Bern, Universität Bern (Prof. Dr. P. Zinsli). - Er- 
hebungen in der Literatur und in historischen Plänen und Karten: Geogr. Institut der Universität Bern, Staatsarchiv Bern, Stadt- und Hochschulbibliothek Bern. - Siegfried-Atlas Blatt 178, Langenthal (1857/1879). - Landeskarte der Schweiz 1:25 000, Blätter 1108 und 1128, Murgenthal und Langenthal (1955).

\section{LA TOPONYMIE DANS LE DÉVELOPPEMENT HISTORIQUE DE LA FORÊT}

On a examiné récemment la composition et l'étendue ancienne de la forêt du point de vue de développement historique. Pour y parvenir, il fallait étudier les noms topographiques de quelques communes bernoises, dont celle de Langenthal. Il est alors indispensable d'examiner l'origine des noms topographiques en consultant les chroniques et les anciennes cartes de la région en question, ainsi que les résultats des recherches dans le domaine des sciences naturelles.

En ce qui concerne la partie inférieure de la vallée de la Langeten, on avait coutume de dire que le défrichage avait été fait surtout entre le loe et le $12 \mathrm{e}$ siècle. Or, il a été prouvé, d'après le cadastre du couvent de St-Urban ainsi que le démontrent lse cartes de l'époque, que le défrichage principales se situerait dans le $14 \mathrm{e}$ et le $15 \mathrm{e}$ siècle. Les chiffres suivantes montrent comment les choses se sont passées par la suite: La surface de la forêt fut, en 1400; d'environ 850 ha, en 1810 de 730 ha et en 1942 encore de 644 ha. Ce sont des chiffres basés sur les cadastres (Urbar) du couvent de St-Urban.

La composition des forêts de cette même région au moyen-âge fut révélée par l'examen des noms topographiques et de leur évolution, ainsi que par l'analyse du pollen. Ces résultats coïncident étonnamment avec ceux obtenus dans une contrée semblable de Thurgovie. Les forêts étudiées dans la région de Langenthal se composent aujourd'hui de $80 \%$ de conifères et de $10 \%$ de hêtres et de chênes. La fréquence des noms topographiques, dérivés de noms d'arbres, permet de dire qu'en 1400 il n'y avait que $10 \%$ de conifères, mais 30 à $40 \%$ de hêtres et de chênes. Pour ce qui en est du bouleau, de l'aune et du saule, les noms topographiques qui en sont dérivés révèlent à peu près la même fréquence que chêne et hêtre.

\section{MALTA \\ Kleine Insel mit großer Vergangenheit WALTER KÜMMERLY}

Mit Farbbild und Landkarte

Inseln sind Berge und Gebirge, die zum Teil aus dem Wasser ragen. Durch Hebung und Senkung des Festlandes und zufolge von Schwankungen der Meeresoberfläche entstehen und verschwinden Halbinseln, Inseln und In:elgruppen. Vor $50000 \mathrm{Jahren}$ soll der Wasserspiegel im Mittelmeer $1000 \mathrm{~m}$ tiefer gestanden sein als heute, zwei Binnenseen lagen westlich und östlich von Calabrien und Sizilien. Italien und Tunis bildeten eine Brücke von Europa nach Afrika, auch die Adria und die Ägäis stellten ausgedehnte Landflächen dar.

Nahe der Marsa Scirocco Bay (Ostküste Marsaxlokk Bay) liegt an der Straße Valletta-Birzebuggia die aufschlußreiche Höhle von Ghar Dalam und das Museum mit entsprechenden Fundstücken. Das $200 \mathrm{~m}$ horizontal sich in den Berg erstreckende Gewölbe ist so reich an Spuren und mit Überresten von Menschen und Tieren angefüllt, daß ein großer Zeitraum der Frühgeschichte Maltas und der Mittelmeerkulturen daraus erforscht werden kann. In vier verschiedenen Bodenschichten eingebettet liegen Scherben alter Töpfereien, menschliche und tierische Knochen. Außerordentlich ist die Entdeckung von menschlichen Gebissen, die dem Neandertaler zugeschrieben werden, und Skelette von Elefanten verschiedener Größe, besonders aber der Zwergelefanten von $1 \mathrm{~m}$ Höhe. Das Alter menschlicher oder tierischer Besiedlung auf der Insel wird auf 50-150 000 Jahre ge:chätzt, und das Vorhandensein der Tiere verschiedener Art bekräftigt die Annahme der Landverbindungen von Italien nach Sizilien und nach Malta.

Das Schmelzwasser der letzten Eiszeit hat das Mittelmeerbecken wieder auf den heutigen Stand gefüllt, Malta ist eine Insel geworden $\left(315.46 \mathrm{~km}^{2}\right)$, die bei günstiger 